\title{
The Steady Loops of Sdof Perfectly Elastoplastic Structures Under Sinusoidal Loadings
}

Chein-Shan Liu

Associate Professor, Department of Mechanical and Marine Engineering, National Taiwan Ocean University, Keelung, Taiwan, R.O.C.

Follow this and additional works at: https://jmstt.ntou.edu.tw/journal

Part of the Engineering Commons

\section{Recommended Citation}

Liu, Chein-Shan (2000) "The Steady Loops of Sdof Perfectly Elastoplastic Structures Under Sinusoidal Loadings," Journal of Marine Science and Technology. Vol. 8: Iss. 1, Article 6.

DOI: $10.51400 / 2709-6998.2453$

Available at: https://jmstt.ntou.edu.tw/journal/vol8/iss1/6

This Research Article is brought to you for free and open access by Journal of Marine Science and Technology. It has been accepted for inclusion in Journal of Marine Science and Technology by an authorized editor of Journal of Marine Science and Technology. 


\section{THE STEADY LOOPS OF SDOF PERFECTLY ELASTOPLASTIC STRUCTURES UNDER SINUSOIDAL LOADINGS}

\begin{abstract}
Keywords: Perfectly elastoplastic structure, Dissipation loop, Elastic shakedown, Limit cycle.

ABSTRACT

The model of single degree of freedom perfectly elastoplastic structures under external loading is treated in this paper. It is analyzed and pointed out that for the perfectly elastoplastic structure the equation of motion is nothing but a two-phase linear system with an on-off switch. Then the exact solutions of the dynamic responses are derived for sinusoidal loadings. For such loading we prove that no matter how large the amplitude is, the structure is impossible undergoing a permanent plastic motion; conversely, the long term behavior exhibits either stable hysteresis loop and limit cycle, or elastic shakedown. A phase plane estimation method of the steady response is developed, which includes not only the formulae for calculating the amplitude of displacement and two time lags, but also the identification of the parameters' values for elastic shakedown and the maximum size of dissipation loop. The accuracy of the formulae is confirmed by comparing with the exact solutions. The mean displacement however depends on the initial conditions, a transient response, not being determined by the steady state estimation.
\end{abstract}

Chein-Shan Liu

\section{INTRODUCTION}

Structures may exhibit linearly elastic behavior under moderately small loading; however, when subjected to severe excitations such as strong earthquake ground motion, they would respond inelastically and exhibit hysteretic behavior. Hysteretic models have been used for the inelastic earthquake-resistant design of structures [1]. Recently, some kinds of nonlinear hysteretic isolators [2-5] have gotten a lot of applications due to their good hysteretic property for the energy dissipation. Both the analytical modeling and response prediction of hysteretic systems have been an area of ever-increasing interest.

It appears that most types of relatively mild

Paper Received May 1, 2000. Author for Correspondence: C.-S. Liu. *Associate Professor, Department of Mechanical and Marine Engineering, National Taiwan Ocean University, Keelung, Taiwan, R.O.C. nonlinearities can be dealt with successfully by equivalent linearization techniques [6-8]. But these techniques do not succeed in capturing the essence of strongly nonlinear hysteretic behavior, i.e., energy dissipated through the development of plastic displacement.

The most commonly used model for describing the nonlinear hysteretic restoring force-displacement behavior is the perfectly elastoplastic system. For the proposed nonlinear models the differential equations of motion are used to analyze the responses of the modeled structures under external loading. Those equations are usually solved by the step-by-step integration techniques. In this paper a rigorously correct formulation of perfectly elastoplastic model is presented and the dynamic responses of such a single degree of freedom (SDOF) elastoplastic structure under external loading is treated and the exact solutions of the responses are derived. Since the exact solutions are available the dynamic characteristics of the perfectly elastoplastic structure are investigated in depth.

Even the perfect elastoplasticity is one of the most important models to assess the structure inelastic behavior under external loading, in the open literature there still lacks a simple method to study the long term behavior in the steady state of the structure under periodic loading. Thus, some important issues such as developing a simple yet effective formula for predicting the oscillation amplitude, the selection of suitable parameters' values for elastic shakedown, and also the best parameters' values for maximizing the size of dissipation loop, are all not yet solved. Therefore, we would propose a phase plane estimation method to resolve these problems.

\section{PERFECTLY ELASTOPLASTIC STRUCTURE}

\section{Equation of Motion}

Let us consider a single degree of freedom (SDOF) structure, schematically shown in Fig. 1, which is subjected to an external loading $p(t)$. The equation of 


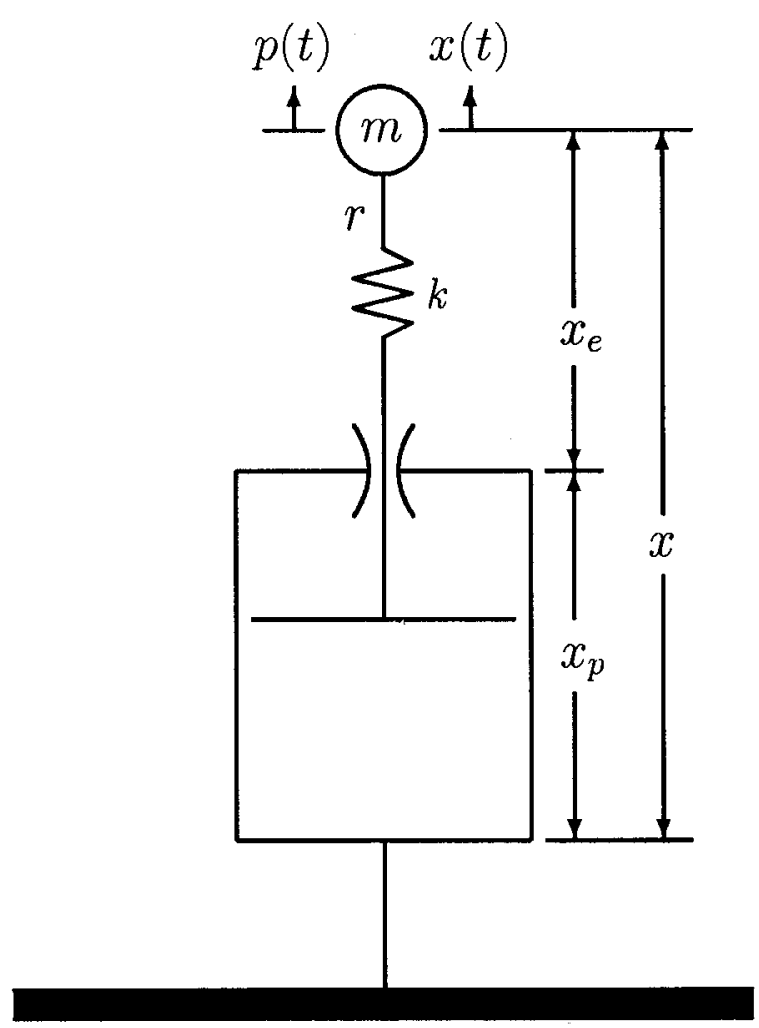

Fig. 1. Perfectly elastoplastic structure subjected to external loading.

motion of the structure can be written as

$$
m \ddot{x}(t)+r(t)=p(t)
$$

which together with some prescribed initial conditions $x\left(t_{i}\right)$ and $\dot{x}\left(t_{i}\right)$ constitute an initial value problem. Here, $t$ is time and $t_{i}$ is an initial time; a superposed dot represents a time differentiation; $m$ and $r$ are the mass and constitutive force of the structure, respectively; and $x(t)$ is the displacement. This oscillator called elasticplastic oscillator comprises three parts: the mass, the slider and the elastic spring.

\section{Perfectly Elastoplastic Model}

In this paper the non-linearity of the structure is reflected in a perfectly elastoplastic model for the relationship of constitutive force and displacement, which is schematically shown in Fig. 2. For perfect elastoplasticity the following postulations are adopted [9]:

$$
\begin{aligned}
& \dot{x}=\dot{x}_{e}+\dot{x}_{p}, \\
& \dot{r}=k \dot{x}_{e},
\end{aligned}
$$

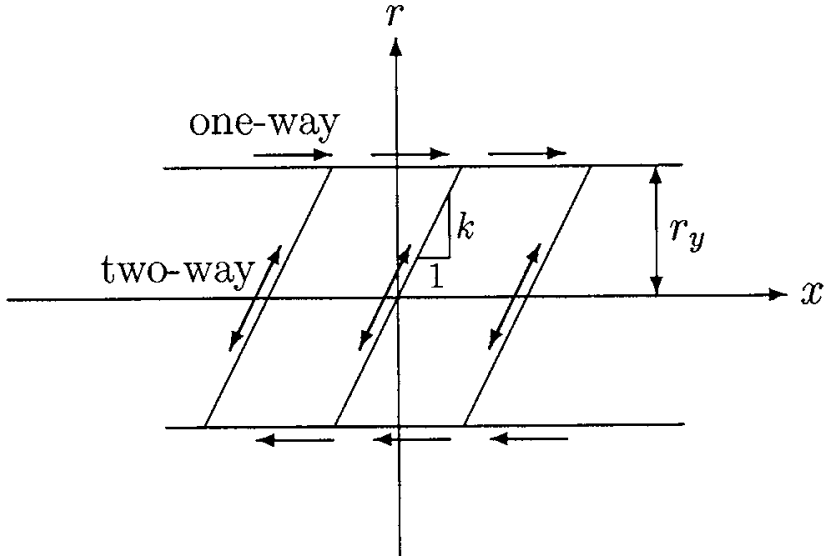

Fig. 2. The relationship of constitutive force and displacement for perfect elastoplasticity.

$$
\begin{aligned}
& \dot{\lambda} r=r_{y} \dot{x}_{p}, \\
& |r| \leq r_{y}, \\
& \dot{\lambda} \geq 0,
\end{aligned}
$$

$|r| \dot{\lambda}=r_{y} \dot{\lambda}$

It is a special case of the bilinear structure model studied in [10]. The two constants, namely the elastic stiffness $k$ and the yield strength $r_{y}$, are assumed to be positive. Here $x, x_{e}, x_{p}$ and $r$ are the displacement, elastic displacement, plastic displacement and constitutive force, respectively; $\lambda$ is a scalar named equivalent plastic displacement, which is closely related to the dissipated energy through the power of dissipation $r_{y} \dot{\lambda}$. All $x, x_{e}, x_{p}, r$, and $\lambda$ are functions of one and the same independent variable, time $t$.

If one replaces the series connection of the slider and the elastic spring in the elastic-plastic oscillator by a parallel connection of that two components, one will obtain a Coulomb oscillator with the friction bound $r_{y}$ : $=m g \mu$, where $g$ is the constant of gravitation and $\mu$ is the dynamic friction coefficient; see, e.g. [11]. However, because the two oscillators have slightly different arrangement of the mechanical components, their behaviors will be seen rather different although they have the same mechanical components and also the same values of the parameters.

\section{Switch of Elasticity and Plasticity}

Combining Eqs. (2)-(4), we have

$$
\dot{r}+\frac{k}{r_{y}} \dot{\lambda} r=k \dot{x} .
$$


The complementary trios (5)-(7) enable the model to possess an elastic-plastic switch, the switching criteria of which are derived right below. The product of $r$ and Eq. (8) is

$$
r \dot{r}+\frac{k}{r_{y}} \dot{\lambda} r^{2}=k r \dot{x}
$$

which, due to the constancy of $r_{y}$ and the positivity of $k$, asserts that

$$
|r|=r_{y} \Rightarrow r_{y} \dot{\lambda}=r \dot{x}
$$

Upon recalling the positivity of $r_{y}$ the following elastic-plastic switching criteria can be proved:

$$
\dot{\lambda}= \begin{cases}\frac{1}{r_{y}} r \dot{x}>0 & \text { if }|r|=r_{y} \text { and } r \dot{x}>0, \\ 0 & \text { if }|r|<r_{y} \text { or } r \dot{x} \leq 0 .\end{cases}
$$

According to the complementary trios (5)-(7), there are just two phases: (i) $\dot{\lambda}>0$ and $|r|=r_{y}$, and (ii) $\dot{\lambda}=0$ and $|r| \leq r_{y}$. From the criteria (11) it is clear that (i) corresponds to the plastic phase while (ii) to the elastic phase.

\section{Two-phase Linear System}

Note that

$$
\text { PLASTIC } \quad r(t)=r\left(t_{i}\right)
$$

in the plastic phase, if $t_{i}$ is chosen to be the switched-on time; hence, Eq. (1) becomes

$$
\text { PLASTIC } m \ddot{x}(t)=p(t)-r\left(t_{i}\right) \text {. }
$$

In the elastic phase, i.e. $\dot{\lambda}=0$, Eq. (4) requires $x_{p}$ be frozen and Eqs. (2) and (3) reduce to $\dot{r}=k \dot{x}$, integrating of which from $t_{i}$ to $t$ yields

$$
\text { ELASTIC } r(t)=r\left(t_{i}\right)+k\left[x(t)-x\left(t_{i}\right)\right],
$$

such that Eq. (1) changes to

$$
\text { ELASTIC } m \ddot{x}(t)+k x(t)=p(t)-r\left(t_{i}\right)+k x\left(t_{i}\right)
$$

During the elastic phase the motion of the elasticplastic oscillator is described by Eqs. (14) and (15), and we call it elastic motion. Conversely, in the plastic phase the motion of the elastic-plastic oscillator is governed by Eqs. (12) and (13), and we call it plastic motion. Indeed, it is a two-phase linear system with an elastic-plastic switch to decide which motion alived in the subsequent time interval.

\section{RESPONSE TO SINUSOIDAL LOADING}

In what follows the driving force is taken to be harmonic with a single driving (angular) frequency $\omega_{d}$,

$$
p(t)=p_{0} \sin \omega_{d} t
$$

where $p_{0}$ is the amplitude of periodic excitation on mass.

\section{Exact Solutions}

For the above input the responses of the elastic motion and plastic motion are derived, respectively, as follows:

\section{(A) Elastic motion:}

$$
\begin{aligned}
x(t) & =x\left(t_{i}\right)+\frac{\dot{x}\left(t_{i}\right)}{\omega_{n}} \sin \omega_{n}\left(t-t_{i}\right)-\frac{r\left(t_{i}\right)}{k}[1 \\
& \left.-\cos \omega_{n}\left(t-t_{1}\right)\right]+A\left[\sin \omega_{d} t-\cos \omega_{n}(t\right. \\
& \left.\left.-t_{i}\right) \sin \omega_{d} t_{i}-r_{w} \sin \omega_{n}\left(t-t_{i}\right) \cos \omega_{d} t_{i}\right],
\end{aligned}
$$

where $\omega_{n}=\sqrt{k / m}$ is the natural angular frequency of the structure,

$$
A:=\frac{p_{0}}{k\left(1-r_{w}^{2}\right)},
$$

and

$$
r_{w}:=\frac{\omega_{d}}{\omega_{n}}>0
$$

is the frequency ratio. The constitutive force $r$ appeared in the above should be supplemented by Eq. (14).

(B) Plastic motion:

$$
\begin{aligned}
x(t) & =x\left(t_{i}\right)+\left[\dot{x}\left(t_{i}\right)+\frac{r\left(t_{i}\right) t_{i}}{m}+\frac{p_{0}}{m \omega_{d}} \cos \omega_{d} t_{i}\right]\left(t-t_{i}\right) \\
& -\frac{p_{0}}{m \omega_{d}^{2}}\left[\sin \omega_{d} t-\sin \omega_{d} t_{i}\right]-\frac{r\left(t_{i}\right)}{2 m}\left(t^{2}-t_{i}^{2}\right)
\end{aligned}
$$

The constitutive force is simply given by $r(t)=$ $r\left(t_{i}\right)$. Unlike the elastic motion, which will be resonant when $r_{w}=1$, the plastic motion as shown in Eq. (20) never occurrs plastic resonance. However, for the bilinear elastoplastic model it may has plastic resonance [10]. 


\section{Transitions between Elastic and Plastic Motions}

The transition points between elastic and plastic motions are determined by the on-off switch criteria derived in Eq. (11).

\subsection{Determination of the onset of plastic motion}

Given the initial values $r\left(t_{i}\right)$ and $\dot{x}\left(t_{i}\right)$ at an initial time $t_{i}$, the transition time from elastic motion to plastic motion can be determined by solving the equation $|r(t)|=r_{y}$, where $r(t)$ is obtained by substituting Eq. (17) into Eq. (14),

$$
\begin{aligned}
r(t) & =r\left(t_{i}\right) \cos \omega_{n}\left(t-t_{i}\right)+\frac{k \dot{x}\left(t_{i}\right)}{\omega_{n}} \sin \omega_{n}\left(t-t_{i}\right) \\
& +k A\left[\sin \omega_{d} t-\cos \omega_{n}\left(t-t_{i}\right) \sin \omega_{d} t_{i}\right. \\
& \left.-r_{\omega} \sin \omega_{n}\left(t-t_{i}\right) \cos \omega_{d} t_{i}\right] .
\end{aligned}
$$

The resulting equation is transcendental in nature so that a numerical method may be invoked to calculate the switch-on time $t=t_{\text {on }}$.

\subsection{Transition from plastic motion to elastic motion}

When a plastic motion interval is switched off at a time moment, that is, $|r|<r_{y}$ or $r \ddot{x} \leq 0$, the structure will be switched to an elastic motion interval according to the switching criteria (11). The end time of the plastic motion is determined by solving $\dot{x}(t)=0$ for $t$ with the following equation:

$$
\begin{aligned}
\dot{x}(t) & =\dot{x}\left(t_{i}\right)-\frac{r\left(t_{i}\right)}{m}\left(t-t_{i}\right)-\frac{p_{0}}{m \omega_{d}}\left[\cos \omega_{d} t-\cos \omega_{d} t_{i}\right] \\
& =0
\end{aligned}
$$

which is also a transcendental equation still requiring a numerical method to calculate the switch-off time $t=$ $t_{\text {off }}$.

Furthermore we can prove that there exists at least one solution $t$ for the equation $\dot{x}(t)=0$. If $\dot{x}\left(t_{i}\right)>0$ we have $r\left(t_{i}\right)=r_{y}>0$ by criteria (11). Thus,

$$
\dot{x}\left(t_{i}\right)-\frac{r\left(t_{i}\right)}{m}\left(t_{1}-t_{i}\right)+\frac{p_{0}}{m \omega_{d}} \cos \omega_{d} t_{i}=\frac{-p_{0}}{m \omega_{d}}
$$

should hold for some $t_{1}>t_{i}$, that is,

$$
t_{1}=t_{i}+\frac{m}{r\left(t_{i}\right)}\left[\dot{x}\left(t_{i}\right)+\frac{p_{0}}{m \omega_{d}} \cos \omega_{d} t_{i}+\frac{p_{0}}{m \omega_{d}}\right] .
$$

At this moment we have

$$
\begin{aligned}
\dot{x}\left(t_{1}\right) & =\dot{x}\left(t_{i}\right)-\frac{r\left(t_{i}\right)}{m}\left(t_{1}-t_{i}\right)-\frac{p_{0}}{m \omega_{d}}\left[\cos \omega_{d} t_{1}-\cos \omega_{d} t_{i}\right] \\
& =-\frac{p_{0}}{m \omega_{d}}\left[1+\cos \omega_{d} t_{1}\right] \leq 0,
\end{aligned}
$$

which together with $\dot{x}\left(t_{i}\right)>0$ implies the conclusion that there exists at least one solution $t$ for $\dot{x}(t)=0$ in the interval $\left(t_{i}, t_{1}\right]$. Similarly, if $\dot{x}\left(t_{i}\right)<0$ we have $r\left(t_{i}\right)=-r_{y}$ $<0$ by criteria (11). Thus,

$$
\dot{x}\left(t_{i}\right)-\frac{r\left(t_{i}\right)}{m}\left(t_{1}-t_{i}\right)+\frac{p_{0}}{m \omega_{d}} \cos \omega_{d} t_{i}=\frac{p_{0}}{m \omega_{d}}
$$

should hold for some $t_{1}>t_{i}$, that is,

$$
t_{1}=t_{i}+\frac{m}{r\left(t_{i}\right)}\left[\dot{x}\left(t_{i}\right)+\frac{p_{0}}{m \omega_{d}} \cos \omega_{d} t_{i}-\frac{p_{0}}{m \omega_{d}}\right] .
$$

At this time $t_{1}$ we have

$$
\begin{aligned}
\dot{x}\left(t_{1}\right) & =\dot{x}\left(t_{i}\right)-\frac{r\left(t_{i}\right)}{m}\left(t_{1}-t_{i}\right)-\frac{p_{0}}{m \omega_{d}}\left[\cos \omega_{d} t_{1}-\cos \omega_{d} t_{i}\right] \\
& =\frac{p_{0}}{m \omega_{d}}\left[1-\cos \omega_{d} t_{1}\right] \geq 0,
\end{aligned}
$$

which together with $\dot{x}\left(t_{i}\right)<0$ implies the conclusion that there exists at least one solution $t$ for $\dot{x}\left(t_{i}\right)=0$ in the interval $\left(t_{i}, t_{1}\right]$. Therefore, we have proved that the considered structure when subjecting to the periodic loading (16) no matter what the amplitude $p_{0}$ and driving frequency $\omega_{d}$ are, will not always stay in the plastic motion.

\section{Responses}

Now by piecing together the above two solutions (17) and (20) of elastic and plastic motions, the exact responses of the elastic-plastic oscillator are obtained. The dynamics of the model depending on the loading history, however, switches between the two motions. Two typical responses are shown in Fig. 3, one for smaller loading amplitude, $p_{0}=100 \mathrm{~N}$, and the other for larger loading amplitude, $p_{0}=250 \mathrm{~N}$. For both cases the driving frequencies are taken to be $\omega_{d}=2 \pi$, and the other parameters are $\omega_{n}=\omega_{d} / 2=\pi \mathrm{rad} / \mathrm{s}, m=10 \mathrm{~N} \mathrm{~s} / \mathrm{cm}$, $k=m \omega_{n}^{2}=10 \pi^{2} \mathrm{~N} / \mathrm{cm}$, and $r_{y}=50 \mathrm{~N}$. The plastic displacement is given by $x_{p}(t)=x_{p}\left(t_{i}\right)+x(t)-x\left(t_{i}\right)$ in the plastic motion, and $x_{p}(t)=x_{p}\left(t_{i}\right)$ in the elastic motion. We set $x_{p}\left(t_{0}\right)=0$ initially. For the first case it can be seen that the response settles down to a stabilized 1/2subharmonic motion with pure elastic motion after the initial plastic motion in the first one and half cycles. Conversely, for the second case it can be seen from Fig. 3(g) that the response tends to a stabilized hysteresis cycle with finite area inside the loop, so that energy is dissipated constantly unless input loading is terminated, and from Fig. 3(h) that in the phase plane 

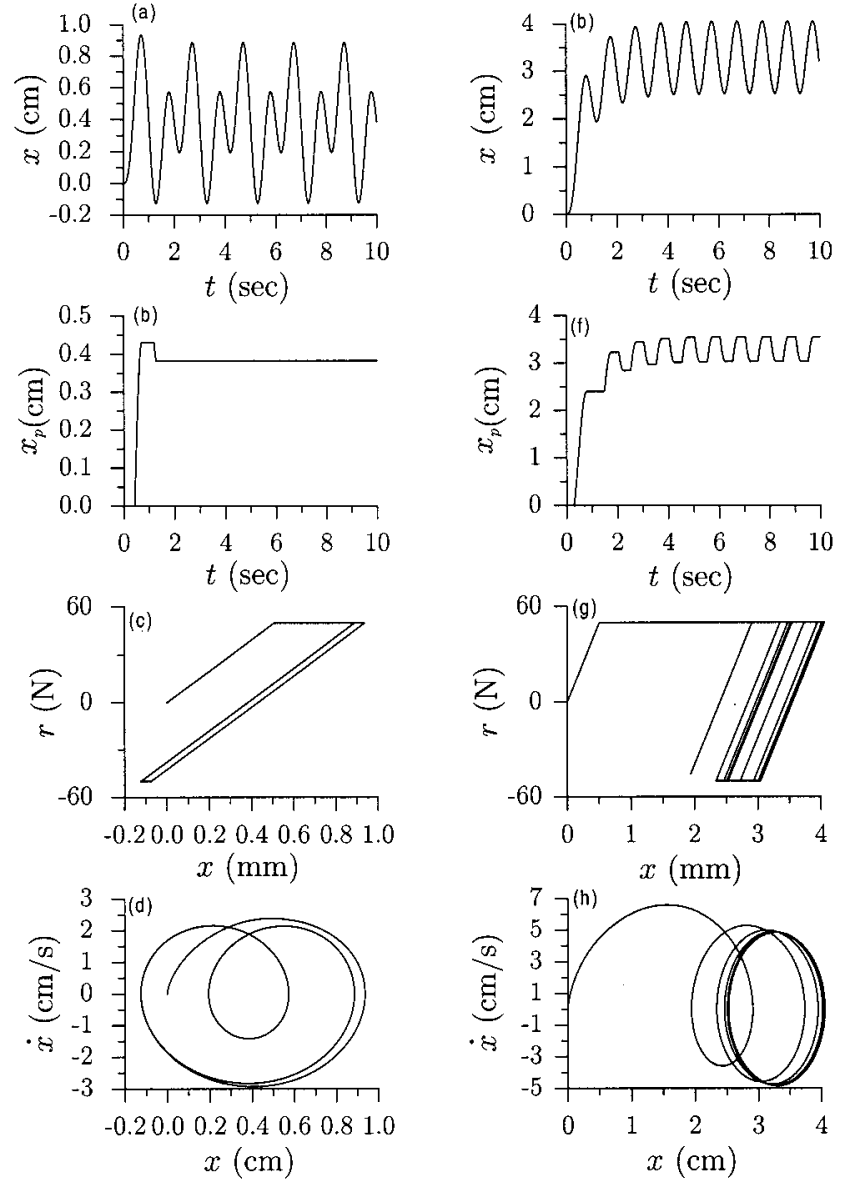

Fig. 3. Two typical responses of the elastic-plastic oscillator with elastic shakedown of (a)-(d) under smaller loading force, $p_{0}=100 \mathrm{~N}$, and steady dissipation loop of (e)-(h) under larger loading force, $p_{0}=$ $250 \mathrm{~N}$.

$(x, \dot{x})$ the orbit tends to a limit cycle. Figure 3(f) shows a typical phase transition of the responses in some time interval, in which $x_{p}$ is constant in the elastic motion and is varying in the plastic motion. From this plot it can be seen that the transitions from one type motion to another type motion occur frequently and regularly. Due to such peculiarity of the switch of the responses the steady state analyses usually employed in the elastic system are no longer suitable for the dynamic analyses of the elastoplastic system, since the frequent switch renders the responses not always stay in one of the two motions. In the next section we are going to study the steady responses via a new phase plane method.

As mentioned earlier although the Coulomb oscillator and the elastic-plastic oscillator both have the same mechanical components, but their arrangement of these components are slightly different. For this reason we may interest on the difference of their behaviors. We compare the behaviors of the two oscillators in Fig. 4
Elastic-plastic oscillator

Coulomb oscillator
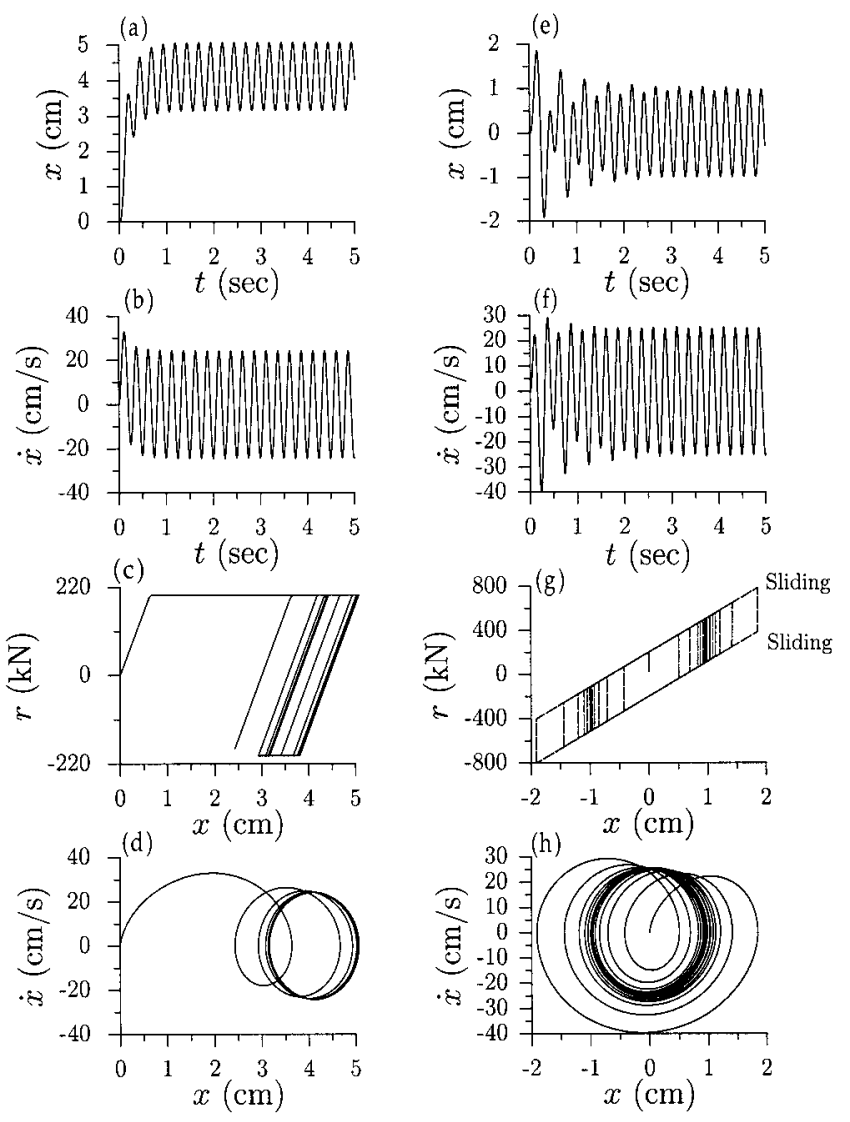

Fig. 4. Comparison of the responses for the elastic-plastic oscillator and the Coulomb oscillator under the same values of the parameters of the mechanical components, the same loading condition and also the same initial conditions.

with the following same parameters: $\omega_{n}=\pi \mathrm{rad} / \mathrm{s}, m=$ $200 \mathrm{kN} \mathrm{s}^{2} / \mathrm{m}, k=m \omega_{n}^{2}=200 \pi^{2} \mathrm{kN} / \mathrm{m}, r_{y}=200 \mathrm{kN}$, and the same loading $p_{0}=1000 \mathrm{kN}$ and $\omega_{d}=2 \omega_{n}=2 \pi \mathrm{rad} /$ $\mathrm{s}$, as well as the same initial conditions $x\left(t_{i}\right)=0$ and $\dot{x}\left(t_{i}\right)$ $=0$. Figs. 4(a)-(d) display the results for the elasticplastic oscillator and Figs. 4(e)-(h) for the Coulomb oscillator. It can be seen that the two oscillators respond very different in every aspects. Especially we call attention to the following points:

(i) The elastic-plastic oscillator has a positive mean displacement oscillating in the positive side $x>0$ with the amplitude slightly smaller than the one of the Coulomb oscillator, whose mean displacement is zero.

(ii) The elastic-plastic oscillator has a constant $r=r_{y}$ $=200 \mathrm{kN}$ during plastic motion, but in the sliding motion of the Coulomb oscillator the constitutive force $r$ is increased up to the value $800 \mathrm{kN}$.

(iii) During sticking phase the displacement of the 


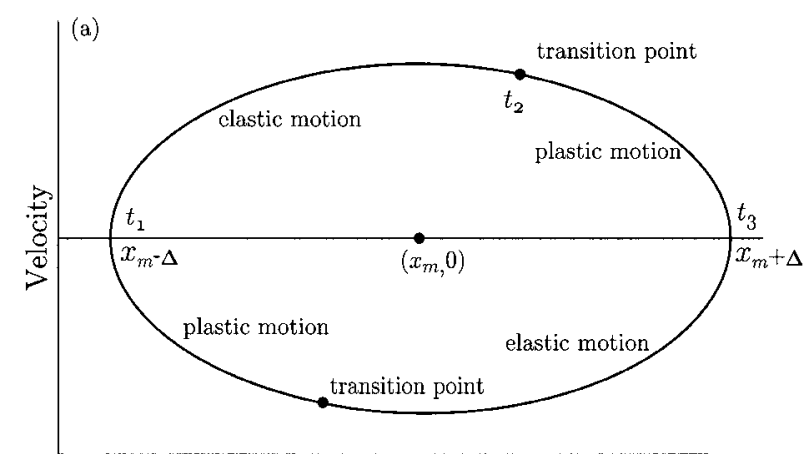

Displacement

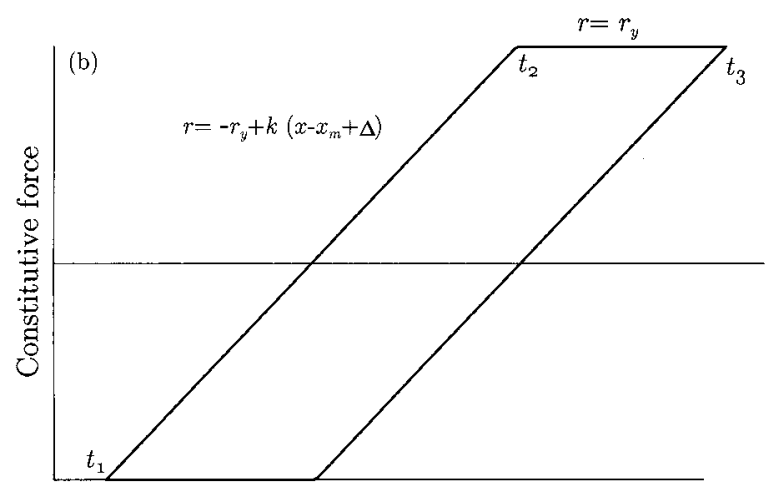

Displacement

Fig. 5. A typical steady-state response with (a) limit cycle in the phase plane of displacement-velocity, (b) dissipation loop in the plane of displacement-constitutive force; $t_{1}, t_{2}$, and $\Delta$ are all to be determined.

Coulomb oscillator is simply given by $x(t)=x\left(t_{i}\right)$, while in the sliding phase the displacement is given by Eq. (17) (see also Eq. (27) in [11]). That is in the sliding phase of the Coulomb oscillator the governing equation of which is the same as the elastic motion of the elastic-plastic oscillator.

(iv) After initial sticking the Coulomb oscillator executes subsequently a sliding-sliding type motion as shown in Fig. 4(g); however, the elastic-plastic oscillator always moves with the manner of elastic motion-plastic motion as shown Fig. 4(c).

\section{ESTIMATION OF THE STEADY RESPONSES}

There are two closed loops as shown in Figs. 3(g) and $3(\mathrm{~h})$, one for the hysteretic loop and the other for the limit cycle. Under what conditions of the parameters and the inputs that the loops exist? Let us consider a steady motion of the elastic-plastic oscillator and assume the phase curve of the steady motion in the phase plane $(x, \dot{x})$ to be symmetrical with respect to the point $\left(x_{m}, 0\right)$, where $x_{m}$ is the mean of the displacement in the steady state. Therefore, the phase curve is closed in the phase plane and it suffices to consider only one half of the curve, say the upper branch. Referring to Fig. 5(a), which is the steady part of Fig. 3(h), let $x_{m}+\Delta$ denotes the maximum displacement of the steady motion, $t_{2}$ the transition time between elastic motion and plastic motion, and $t_{1}$ the starting time of elastic motion, such as to match the exact solutions of the steady response of the elastic-plastic oscillator. Because of the periodicity of the input and the symmetry with respect to $\left(x_{m}, 0\right)$ of the steady motion, we may assume

$$
t_{3}=t_{1}+\frac{\pi}{\omega_{d}}
$$

and estimate the three parameters, namely the amplitude of displacement $\Delta$, and the time lags $t_{1}$ and $t_{2}$.

\section{An Estimation of the Steady Loops}

In the time interval $t_{1} \leq t \leq t_{2}$, the constitutive force in view of Fig. 5(b) (the steady part of Fig. 3(g)) is given by

$$
r=-r_{y}+k\left(x-x_{m}+\Delta\right) \text {. }
$$

Substituting it into Eq. (1) we obtain

$$
m \ddot{x}(t)+k x(t)=r_{y}+k\left(x_{m}-\Delta\right)+p_{0} \sin \omega_{d} t,
$$

whose general solution is given by

$$
\begin{aligned}
x_{1}(t) & =\mathrm{A} \sin \omega_{d} t+x_{y}+x_{m}-\Delta+a_{1} \sin \omega_{n}\left(t-t_{1}\right) \\
& +b_{1} \cos \omega_{n}\left(t-t_{1}\right),
\end{aligned}
$$

where

$$
x_{y}:=\frac{r_{y}}{k}
$$

is the yield displacement. In the above $x_{m}$ is the mean drift of the displacement; see Fig. 5(a). Similarly, in the time interval $t_{2} \leq t \leq t_{3}$ we have

$$
x_{2}(t)=B \sin \omega_{d} t-\frac{r_{y}}{2 m}\left(t-t_{3}\right)^{2}+a_{2}\left(t-t_{3}\right)+b_{2},
$$

where

$$
B:=\left(1-\frac{1}{r_{w}^{2}}\right) A=-\frac{p_{0}}{k r_{w}^{2}} .
$$

The four constants $a_{1}, b_{1}, a_{2}$ and $b_{2}$ can be determined using the following four conditions:

$$
x_{1}\left(t_{1}\right)=x_{m}-\Delta, \dot{x}_{1}\left(t_{1}\right)=0, x_{2}\left(t_{3}\right)=x_{m}+\Delta, \dot{x}_{2}\left(t_{3}\right)=0 .
$$

As a result we obtain 


$$
\begin{aligned}
x_{1}(t) & =A\left[\sin \omega_{d} t-r_{w} \cos \omega_{d} t_{1} \sin \omega_{n}\left(t-t_{1}\right)\right] \\
& -\left(x_{y}+\mathrm{A} \sin \omega_{d} t_{1}\right) \cos \omega_{n}\left(t-t_{1}\right)+x_{y}+x_{m}-\Delta, \\
x_{2}(t) & =B\left[\sin \omega_{d} t-\sin \omega_{d} t_{3}-\omega_{d}\left(t-t_{3}\right) \cos \omega_{d} t_{3}\right] \\
& -\frac{r_{y}}{2 m}\left(t-t_{3}\right)^{2}+x_{m}+\Delta .
\end{aligned}
$$

Both the constitutive force $r(t)$ and the external force $p(t)$ are continuous at the transition time $t_{2}$ from the elastic motion to the plastic motion, which due to Eq. (1) renders that the acceleration $\ddot{x}(t)$ is also continuous at this transition time $t_{2}$. The continuities of $\dot{x}$ and $x$ are obvious by integrating $\ddot{x}$ and $\dot{x}$, respectively. Thus with Eqs. (30), (31) and (23) the following three conditions:

$$
x_{1}\left(t_{2}\right)=x_{2}\left(t_{2}\right), \quad \dot{x}_{1}\left(t_{2}\right)=\dot{x}_{2}\left(t_{2}\right), \ddot{x}_{1}\left(t_{2}\right)=\dot{x}_{2}\left(t_{2}\right)
$$

become the following three equations:

$$
\begin{gathered}
(A-B) \sin \omega_{d} t_{2}-\left(\mathrm{A} \sin \omega_{d} t_{1}+x_{y}\right) \cos \omega_{n}\left(t_{2}-t_{1}\right) \\
-A r_{w} \cos \omega_{d} t_{1} \sin \omega_{n}\left(t_{2}-t_{1}\right)+x_{y}-B \sin \omega_{d} t_{1} \\
-B \omega_{d}\left(t_{2}-t_{1}-\pi / w_{d}\right) \cos \omega_{d} t_{1}+\frac{r_{y}}{2 m}\left(t_{2}-t_{1}\right. \\
\left.-\pi / w_{d}\right)^{2}-2 \Delta=0 \\
(A-B) \cos \omega_{d} t_{2}+\frac{1}{r_{w}}\left(A \sin \omega_{d} t_{1}+x_{y}\right) \sin \omega_{n}\left(t_{2}\right. \\
\left.\quad-t_{1}\right)-A \cos \omega_{d} t_{1} \cos \omega_{n}\left(t_{2}-t_{1}\right)-B \cos \omega_{d} t_{1} \\
\quad+\frac{r_{y}}{m \omega_{d}}\left(t_{2}-t_{1}-\pi / \omega_{d}\right)=0 \\
(B-A) r_{w}^{2} \sin \omega_{d} t_{2}+\left(A \sin \omega_{d} t_{1}+x_{y}\right) \cos \omega_{n}\left(t_{2}\right. \\
\left.\quad-t_{1}\right)+A r_{w} \cos \omega_{d} t_{1} \sin \omega_{n}\left(t_{2}-t_{1}\right)+x_{y}=0 .
\end{gathered}
$$

In principle, we can combine Eqs. (33)-(35) together utilizing numerical method to determine the three unknowns $t_{1}, t_{2}$, and $\Delta$. However, these equations are too complicated to give us proper information about $t_{1}$, $t_{2}$, and $\Delta$ and their influence factors. We below put these equations into a more concise form, such that in terms of the identified parameters the closed-form representations of $t_{1}, t_{2}$, and $\Delta$ are available. Thus further analyses can be proceeded.

\section{Formula for the Amplitude of Displacement}

Adding Eqs. (33) and (35), we obtain

$$
\begin{aligned}
2 \Delta & =-B\left(\sin \omega_{d} t_{1}+\sin \omega_{d} t_{2}\right) \\
& -B \omega_{d}\left(t_{2}-t_{1}-\pi / \omega_{d}\right) \cos w_{d} t_{1} \\
& +\frac{r_{y}}{2 m}\left(t_{2}-t_{1}-\pi / \omega_{d}\right)^{2}+2 x_{y},
\end{aligned}
$$

where $(A-B)\left(1-r_{w}^{2}\right)=-B$ has been used. Upon the solutions of $t_{1}$ and $t_{2}$ are available, from the above formula $\Delta$ can be determined. In fact, Eqs. (34) and (35) can be used to calculate $t_{1}$ and $t_{2}$ numerically. However, for these equations if we replace $\cos \omega_{d} t_{2}$ and $\sin \omega_{d} t_{2}$, respectively, by

$$
\begin{aligned}
\cos \omega_{d} t_{2} & =\cos \omega_{d}\left(t_{2}-t_{1}\right) \cos \omega_{d} t_{1} \\
& -\sin \omega_{d}\left(t_{2}-t_{1}\right) \sin \omega_{d} t_{1}, \\
\cos \omega_{d} t_{2} & =\sin \omega_{d}\left(t_{2}-t_{1}\right) \cos \omega_{d} t_{1} \\
& +\cos \omega_{d}\left(t_{2}-t_{1}\right) \sin \omega_{d} t_{1},
\end{aligned}
$$

we obtain the simultaneous equations for $\cos \omega_{d} t_{1}$ and $\sin \omega_{d} t_{1}$ as follows:

$$
\begin{aligned}
& a \cos \omega_{d} t_{1}+b \sin \omega_{d} t_{1}=c, \\
& b \cos \omega_{d} t_{1}+e \sin \omega_{d} t_{1}=f
\end{aligned}
$$

where

$$
\begin{aligned}
& a:=r_{w}^{2}+r_{w}^{2} \cos \omega_{n}\left(t_{2}-t_{1}\right)-\left[1+\cos \omega_{d}\left(t_{2}-t_{1}\right)\right] \\
& b:=\sin \omega_{d}\left(t_{2}-t_{1}\right)-r_{w} \sin \omega_{n}\left(t_{2}-t_{1}\right) \\
& c:=\frac{r_{w}\left(1-r_{w}^{2}\right)}{r_{f}}\left[\sin \omega_{n}\left(t_{2}-t_{1}\right)+\omega_{n}\left(t_{2}-t_{1}-\pi / \omega_{d}\right)\right] \\
& e:=\cos \omega_{d}\left(t_{2}-t_{1}\right)-\cos \omega_{n}\left(t_{2}-t_{1}\right) \\
& f:=\frac{1-r_{w}^{2}}{r_{f}}\left[1+\cos \omega_{n}\left(t_{2}-t_{1}\right)\right]
\end{aligned}
$$

and

$$
r_{f}:=\frac{p_{0}}{r_{y}}>0
$$

is the force ratio. Solving $\cos \omega_{d} t_{1}$ and $\sin \omega_{d} t_{1}$ from Eq. (39) and substituting them into the identity $\cos ^{2} \omega_{d} t_{1}+$ $\sin ^{2} \omega_{d} t_{1}=1$, we obtain a single equation as follows:

$$
(c e-b f)^{2}+(a f-b c)^{2}=\left(a e-b^{2}\right)^{2} .
$$

Solve $t_{2}-t_{1}$ using this equation and solve $t_{1}$ using 
the following equation:

$$
t_{1}=\frac{1}{\omega_{d}} \arctan \frac{a f-b c}{c e-b f}
$$

due to Eq. (39). Here the admissible range of $\omega_{d} t_{1}$ is $[0$, $\pi)$. Then substituting the above two results into Eq. (36) we obtain $\Delta$.

In terms of $x_{y}, r_{f}$ and $r_{w}, \Delta$ can be expressed as

$$
\begin{aligned}
2 \Delta & =\frac{x_{y} r_{f}}{r_{w}^{2}}\left[\sin \omega_{d} t_{1}+\sin \omega_{d} t_{2}+\omega_{d}\left(t_{2}-t_{1}\right.\right. \\
& \left.\left.-\pi / \omega_{d}\right) \cos \omega_{d} t_{1}\right]+\frac{x_{y} \omega_{n}^{2}}{2}\left(t_{2}-t_{1}-\pi / \omega_{d}\right)^{2} \\
& +2 x_{y} .
\end{aligned}
$$

We are interest to know the variation of $\Delta$ with respect to $r_{f}$ as well as $r_{w}$ for some fixed value of $x_{y}$. For this purpose let

$$
y:=\omega_{d}\left(t_{2}-t_{1}\right), \quad 0<y \leq \pi
$$

be the independent variable, and thus by solving Eq. (46) for $r_{f}$ we obtain

$$
r_{f}=\sqrt{\frac{(c e-b f)^{2}+(a f-b c)^{2}}{\left[a e-b^{2}\right]^{2}}},
$$

where the nondimensionalized coefficients are now read as

$$
\begin{aligned}
& a:=r_{w}^{2}+r_{w}^{2} \cos \frac{y}{r_{w}}-(1+\cos y), \\
& b:=\sin y-r_{w} \sin \frac{y}{r_{w}} \\
& c:=r_{w}\left(1-r_{w}^{2}\right)\left[\sin \frac{y}{r_{e}}+\frac{y}{r_{w}}-\frac{\pi}{r_{w}}\right] \\
& e:=\cos y-\cos \frac{y}{r_{w}}, \\
& f:=\left(1-r_{w}^{2}\right)\left(1+\cos \frac{y}{r_{w}}\right)
\end{aligned}
$$

Notice that

$$
\begin{cases}r_{f} \rightarrow \infty & \text { if } y \rightarrow 0, \\ r_{f}=\left|1-r_{w}^{2}\right| & \text { if } y=\pi .\end{cases}
$$

From Eqs. (48), (37)-(39) and (49) it follows that

$$
\begin{aligned}
\frac{\Delta}{x_{y}} & =\frac{1}{2 r_{w}^{2}}(1+\cos y) \frac{a f-b c}{a e-b^{2}}+\frac{1}{2 r_{w}^{2}}(y+\sin y \\
& -\pi) \frac{c e-b f}{a e-b^{2}}+\frac{1}{4 r_{w}^{2}}(y-\pi)^{2}+1 .
\end{aligned}
$$
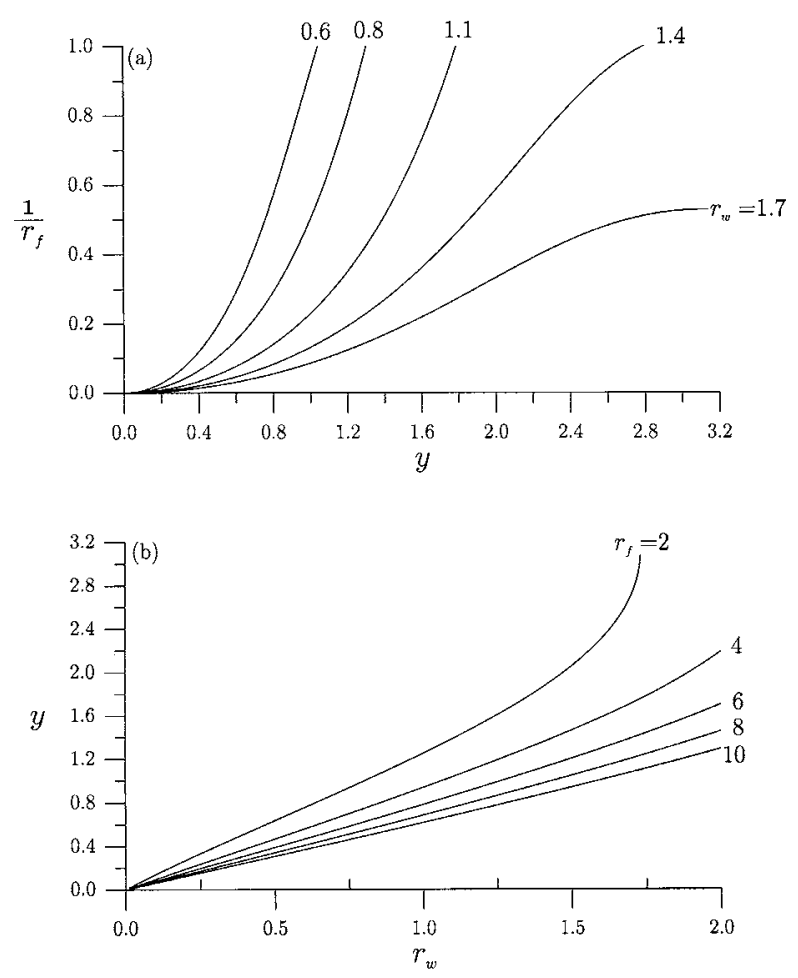

Fig. 6. The force ratio $r_{f}$ is homeomorphic with $y$ when $r_{w}$ is fixed; we plot the variation for five values of $r_{w}$ in (a). Conversely, when $r_{f}$ is fixed we plot the variation of $r_{w}$ versus $y$ for five values of $r_{f}$ in (b).

For fixed value of $r_{w}$, the variation of $\Delta / x_{y}$ with respect to $r_{f}$ can be evaluated as follows: First, it can be seen that $\Delta / x_{y}$ depends on two parameters $r_{w}$ and $y$. Second, for each $r_{w}$ fixed we establish the relation between $y$ and $r_{f}$ via Eq. (50). This relation is an homeomorphism between $r_{f}$ and $y$, since $d\left(1 / r_{f}\right) / d_{y}>0$. For example, in Fig. 6(a) we show some curves of $1 / r_{f}$ with respect to $y$ for $r_{w}=0.6,0.8,1.1,1.4$ and 1.7. The homeomorphism between $r_{f}$ and $y$ renders us very easily to construct the response curves as shown in Fig. 7(a) for $r_{w}=0.6,0.8,1.1,1.4$ and 1.7. Conversely, we fix $r_{f}$ and search the relation between $r_{w}$ and $y$. Similarly, due to the good property of homeomorphism, one and only one solution of $y$ exists for each $r_{w}$, and so it is easy by directly applying the Newton-Raphson numerical method to Eq. (50) to depict the relation between $r_{w}$ and $y$; for example, in Fig. 6(b) we show some curves of $r_{w}$ versus $y$ for $r_{f}=2,4,6,8$ and 10. In Fig. 7(b) the variation of $\Delta / x_{y}$ with respect to $r_{w}$ are plotted for $r_{f}=2$, $4,6,8$ and 10 . In order to assess the validity of formula (57) we also compare the results calculated by it with the exact results calculated by the exact solutions as shown in Eqs. (17) and (20) for different $r_{w}$ 's in Fig. 7(a), and for different $r_{f}$ 's in Fig. 7(b). From these two plots we confirm that formula (57) is very accurate 

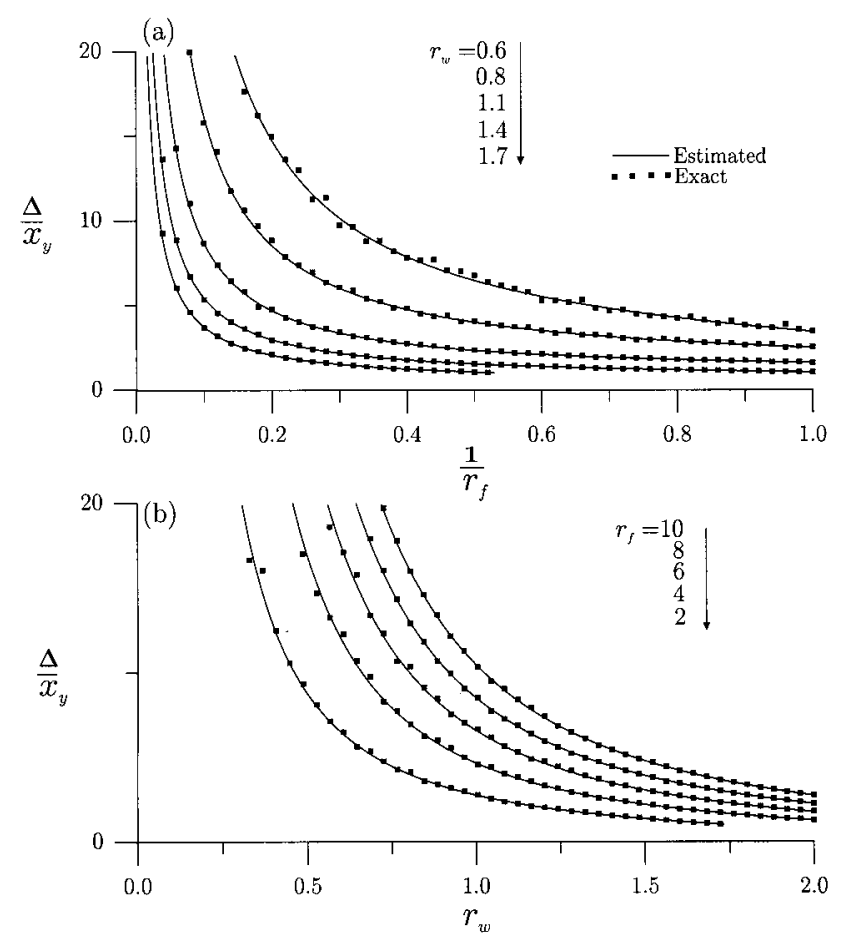

Fig. 7. (a) The variation of $x_{y} / \Delta$ with respect to the force ratio $r_{f}$ for five values of $r_{w}$; (b) The variation of $x_{y} / \Delta$ with respect to the frequency ratio $r_{w}$ for five values of $r_{f}$.

to calculate the oscillating amplitude of the elasticplastic oscillator in the steady state.

\section{Dissipation Loop Size}

For the engineering benefit we may concern the energy dissipation capacity of the considered structure. From Fig. 5(b) the area of energy dissipation loop is found to be

$$
\ell_{a}=4 r_{y}\left(\Delta-x_{y}\right)
$$

and thus with the help of Eq. (57) we can assess the influence of the two control parameters $r_{f}$ and $r_{w}$ on $\ell_{a}$. For this purpose let us introduce the following two nondimensionalized variables for the size of the dissipation loop:

$$
\begin{aligned}
& \Lambda_{1}:=\frac{k \ell_{a}}{p_{0}^{2}}=\frac{4}{r_{f}^{2}}\left(\frac{\Delta}{x_{y}}-1\right), \\
& \Lambda_{2}:=\frac{m \omega_{d}^{2} \ell_{a}}{p_{0}^{2}}=\frac{4 r_{w}^{2}}{r_{f}^{2}}\left(\frac{\Delta}{x_{y}}-1\right) .
\end{aligned}
$$

Some remarks are given in order for the above two formulae: (i) The left-hand side of the first equation

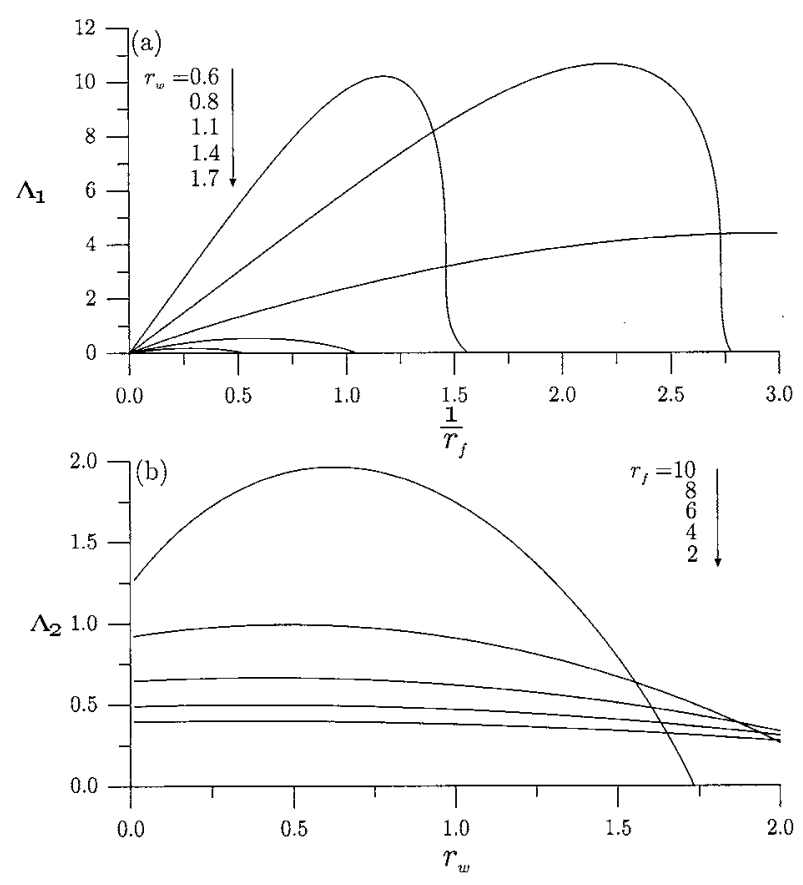

Fig. 8. (a) The variation of the normalized dissipation loop size $\Lambda_{1}$ with respect to the force ratio $r_{f}$ for five values of $r_{w}$; (b) The variation of the normalized dissipation loop size $\Lambda_{2}$ with respect to the frequency ratio $r_{w}$ for five values of $r_{f}$.

may be understood as the dissipation per unit elastic energy, since $p_{0} / k$ is the static displacement of the elastic response and $p_{0}^{2} / k$ is the elastic energy. (ii) The term $\Delta / x_{y}-1$ on the right-hand side of the first equation decreases with respect to $1 / r_{f}$ for each $r_{w}$ as shown in Fig. 7(a); conversely, the term $1 / r_{f}^{2}$ increases with respect to $1 / r_{f}$. Therefore, there exists a best $r_{f}$ to maximize $\Lambda_{1}$ for each $r_{w}$. (iii) Assuming $m \omega_{d}^{2}:=k_{d}$, the left-hand side of the second equation may be written as $\ell_{a} /\left(p_{0}^{2} / k_{d}\right)$; corresponding to (i), it may be understood as the dissipation per pseudo elastic energy with a pseudo elastic stiffness $k_{d}$. (iv) The term $\Delta / x_{y}-1$ on the right-hand side of the second equation is known to be decreased with respect to $r_{w}$ for each $r_{f}$ as shown in Fig. 7(b); nevertheless, the term $r_{w}^{2}$ is increased with respect to $r_{w}$. Hence, there exists one best $r_{w}$ to maximize $\Lambda_{2}$ for each $r_{f}$.

Equation (59) is used to investigate the variation of the nondimensionalized size of the dissipation loop with respect to $r_{f}$, when $k, p_{0}$ and $r_{w}$ are fixed, that is, the influence of the yield strength $r_{y}$ on the size of the dissipation loop. In Fig. 8(a) the variations of $k \ell_{a} / p_{0}^{2}$ with respect to $1 / r_{f}$ are plotted for $r_{w}=0.6,0.8,1.1$, 1.4 and 1.7. For the isolation purpose of the building structure we usually choose the best $r_{f}$ to maximize the dissipation loop size. Under this $r_{f}$ the isolator will achieve the best performance to dissipate as much en- 
ergy as it can. Similarly, Eq. (60) is used to investigate the variation of the nondimensionalized size of the dissipation loop with respect to $r_{w}$, when $m, p_{0}, \omega_{d}$ and $r_{y}$ are kept constants, that is, the influence of the elastic stiffness $k$ on the dissipation loop. In Fig. 8(b) the variations of $m \omega_{d}^{2} \ell_{a} / p_{0}^{2}$ with respect to $r_{w}$ are plotted for $r_{f}=2,4,6,8$ and 10. For the isolation purpose we may choose the best $r_{w}$ to maximize the dissipation loop size.

\section{Elastic Shakedown}

In the case of cyclic loadings the magnitude of the input is not merely a factor characterizing the structural safety. These loads may also result in alternating plastic displacement increment, after a sufficient number of cycles lead to low cycle fatigue and cause the failure of the structure. Therefore, the structural safety requires that the plastic displacement increments due to consecutive load changes should eventually cease, the structural response of further cycles being fully elastic. Such a stabilization of plastic displacements is called (elastic) shakedown or adaptation. Thus, for the purpose of safety we may hope the structure tend to elastic shakedown gradually, without induces further plastic deformation to cause failure of the structure. This requires that the plastic dissipating loop as shown in Fig. 5(a) disappears, which is equivalent to the requirement that $t_{3}=t_{2}=t_{1}+\pi / \omega_{d}$ or $y=\pi$ via Eq. (49). Under this condition by Eqs. (57) and (58) it is obvious that

$$
\Delta=x_{y}, \ell_{a}=0
$$

the latter of which indicates that the size of the dissipation loop is zero. Moreover, under this condition we have

$$
r_{f}=\left|1-r_{w}^{2}\right|
$$

as noted in Eq. (56), and the two time lags are reduced to $t_{1}=\pi /\left(2 \omega_{d}\right)$ and $t_{2}=3 \pi /\left(2 \omega_{d}\right)$. The right-hand side of Eq. (56) $)_{2}$ supplies a lower bound of $r_{f}$ for elastic shakedown. The loading with its $r_{f}$ smaller than $\left|1-r_{w}^{2}\right|$ will tend to elastic shakedown more quicker than the one with the critical value $r_{f}=\left|1-r_{w}^{2}\right|$. Thus, a criterion for elastic shakedown is obtained as follows:

$$
r_{f}=\left|1-r_{w}^{2}\right|
$$

The determination of the control parameters allowing for the adaptation is the main goal of the plasticity theory of shakedown. Here, Eq. (62) gives a constraint for the control parameters, under which the steady response is elastic shakedown. In Fig. 3 the two types of motion, shakedown and not shakedown, have been compared.

\section{CONCLUDING REMARKS}

This paper gaven a correct perfectly elastoplastic model for the relation of constitutive force and displacement, and obtained the exact solutions of the SDOF perfectly elastoplastic structure. However, in order to obtain the whole responses in the course of external loading elastic and plastic motions must be pieced sequentially. The notions of complementary trios and switch in the elastoplastic structural system made the formulation precise and accurate. It was found that the equations of motion for the perfectly elastoplastic structure are a two-phase linear system with an on-off switch. Although by itself sufficing to describe the linear plant of the equation of motion, the state $x$ and $\dot{x}$ must be supplemented by the constitutive force $r$ for a complete state space description of the elastoplastic system which contains the two-phase linear plant subjected to external excitations.

A new estimate was developed for the steady-state oscillation responses; the resulting formula (57) can be used to determine the oscillating amplitude of displacement. It supplied very good results when compared with the exact displacement oscillating amplitude. The formulae for two nondimensionalized sizes of the dissipation loop were derived. It is found that for a fixed frequency ratio there exists a value of the best driving force amplitude to maximize the dissipation loop size. The closed-form formula (62) of the criterion for elastic shakedown was derived. In view of the detrimental effect of alternating plasticity on the structure, the simple formula must be very useful for engineers to select a minimum driving force amplitude to prevent the structure oscillating in the plastic range.

\section{REFERENCES}

1. Newmark, N. M. and Hall, J. W., "Earthquake Spectra and Design," Monograph published by the Earthquake Engineering Research Institute, Los Angeles (1982).

2. Hwang, J. S. and L. H. Shen, "Equivalent elastic seismic analysis of base isolated bridges with lead rubber bearings," Engng. Struct., Vol. 16, pp. 201-209 (1994).

3. Hwang, J. S., Shen, L. H. and J. H. Gates, "Practical analysis of base-isolated bridges with bi-linear hysteresis characteristics," Earthq. Spectra, Vol. 10, pp. 705727 (1994).

4. Robinson, W. H., "Lead-rubber hysteretic bearing suitable for protecting structures during earthquake," Earthq. Engng. Struc. Dyna., Vol. 10, pp. 593-604 (1982).

5. Turkington, D. H. and A. J. Carr, "Development of a design procedure for bridge on lead rubber bearings," 
Engng. Struc., Vol. 11, pp. 2-8 (1989).

6. Caughey, T. K., "Equivalent linearization techniques," J. Acou. Soc. Amer., Vol. 35, pp. 1706-1711 (1963).

7. Iwan, W. D., "A generalization of the concept of equivalent linearization," Int. J. Non-linear Mech., Vol. 8, pp. 279-287 (1973).

8. Nayfeh, A. H. and D. T. Mook, Nonlinear Oscillations, Wiley, New York (1979).

9. Hong, H.-K. and Liu, C.-S., "Prandtl-Reuss elastoplasticity: on-off switch and superposition formulae," Int. J. Solids Struct., Vol. 34, pp. 4281-4304 (1997).

10. Liu, C.-S., "Exact solutions and dynamic responses of SDOF bilinear elastoplastic structures," J. Chinese Inst. Engineers, Vol. 20, pp. 511-525 (1997).

11. Hong, H.-K. and Liu, C.-S., "Coulomb friction oscillator: modelling and responses to harmonic loads and base excitations," J. Sound Vib., Vol. 229, pp. 1171-1192 (2000).

\section{單自由度彈性完全塑性結構在正弦 波荷載下的穞態圈}

$$
\text { 劉進 賢 }
$$

國立臺灣海洋大學機械與輪機工程學系副教授

$$
\text { 摘 要 }
$$

本文探討單自由度彈性完全塑性結構在外加動 態荷載下的數學模式, 推導得出塑性不可逆開關的充 分必要條件。經過深入的分析研究, 本文指出描述單 自由度彈性完全塑性結構的運動方程, 可以轉換成兩 相的線性微分方程及一個開關機制。因此任何外加正 弦波荷載下結構的動態響應可以完全由求得的正解進 行分析及計算。在正弦波荷載下本文證明彈性完全塑 性結構不可能永遠是塑性運動, 反之, 其長時間行爲 應是穞定的遲滞圈、極限環或者是彈性安定。本文提 出一個新的相平面法估測穞態解, 可以計算位移的最 大振幅及兩個時間差, 並定出彈塑性結構會彈性安定 及最大耗散的參數條件。估测的穞態解和由數值計算 的正解進行比較, 發現本文的方法非常好, 幾乎給出 完全相同的結果。 